\title{
Integral Inequalities of Hermite-Hadamard Type for Functions Whose 3rd Derivatives Are s-Convex
}

\author{
Ling Chun ${ }^{1}$, Feng Qi ${ }^{2}$ \\ ${ }^{1}$ College of Mathematics, Inner Mongolia University for Nationalities, Tongliao, China \\ ${ }^{2}$ Department of Mathematics, School of Science, Tianjin Polytechnic University, Tianjin, China \\ Email: chunling1980@qq.com, qifeng618@gmail.com
}

Received September 2, 2012; revised October 2, 2012; accepted October 10, 2012

\begin{abstract}
In the paper, the authors find some new inequalities of Hermite-Hadamard type for functions whose third derivatives are $s$-convex and apply these inequalities to discover inequalities for special means.
\end{abstract}

Keywords: Integral Inequality; Hermite-Hadamard’s Integral Inequality; s-Convex Function; Derivative; Mean

\section{Introduction}

The following definition is well known in the literature.

Definition 1.1. A function $f: I \subseteq \mathbb{R}=(-\infty, \infty) \rightarrow \mathbb{R}$ is said to be convex if

$$
f(\lambda x+(1-\lambda) y) \leq \lambda f(x)+(1-\lambda) f(y)
$$

holds for all $x, y \in I$ and $\lambda \in[0,1]$.

In $[1,2]$, among others, the concepts of so-called quasiconvex and $s$-convex functions in the second sense was introduced as follows.

Definition 1.2 ([1]). A function

$f: I \subseteq \mathbb{R} \rightarrow \mathbb{R}_{0}=[0, \infty)$ is said to be quasi-convex if

$$
f(\lambda x+(1-\lambda) y) \leq \sup \{f(x), f(y)\}
$$

holds for all $x, y \in I$ and $\lambda \in[0,1]$.

Definition 1.3 ([2]). Let $s \in(0,1]$. A function $f: \mathbb{R}_{0} \rightarrow \mathbb{R}_{0}$ is said to be $s$-convex in the second sense if

$$
f(\lambda x+(1-\lambda) y) \leq \lambda^{s} f(x)+(1-\lambda)^{s} f(y)
$$

for all $x, y \in I$ and $\lambda \in[0,1]$.

If $f: I \subseteq \mathbb{R} \rightarrow \mathbb{R}$ is a convex function on $[a, b]$ with $a, b \in I$ and $a<b$, Then we have Hermite-Hardamard's inequality

$$
f\left(\frac{a+b}{2}\right) \leq \frac{1}{b-a} \int_{a}^{b} f(x) \mathrm{d} x \leq \frac{f(a)+f(b)}{2} .
$$

Hermite-Hadamard inequality (1.1) has been refined or generalized for convex, $s$-convex, and quasi-convex functions by a number of mathematicians. Some of them can be reformulated as follows.

Theorem 1.1 ([3, Theorems 2.2 and 2.3]). Let

$f: I^{\circ} \subset \mathbb{R} \rightarrow \mathbb{R}$ be a differentiable mapping on $I^{\circ}$, $a, b \in I^{\circ}$ with $a<b$.
(1) If $\left|f^{\prime}(x)\right|$ is convex on $[a, b]$, then

$$
\begin{aligned}
& \left|\frac{f(a)+f(b)}{2}-\frac{1}{b-a} \int_{a}^{b} f(x) \mathrm{d} x\right| \\
& \leq \frac{(b-a)\left(\left|f^{\prime}(a)\right|+\left|f^{\prime}(b)\right|\right)}{8}
\end{aligned} .
$$

(2) If the new mapping $\left|f^{\prime}(x)\right|^{p /(p-1)}$ is convex on $[a, b]$ for $p>1$, then

$$
\begin{aligned}
& \left|\frac{f(a)+f(b)}{2}-\frac{1}{b-a} \int_{a}^{b} f(x) \mathrm{d} x\right| \\
& \leq \frac{b-a}{2(p+1)^{1 / p}}\left(\frac{\left|f^{\prime}(a)\right|^{p /(p-1)}+\left|f^{\prime}(b)\right|^{p /(p-1)}}{2}\right)^{1-1 / p} .
\end{aligned}
$$

Theorem 1.2 ([4, Theorems 1 and 2]). Let $f: I \subset \mathbb{R} \rightarrow \mathbb{R}$ be a differentiable function on $I^{\circ}$ and $a, b \in I$ with $a<b$, and let $q \geq 1$. If $\left|f^{\prime}(x)\right|^{q}$ is convex on $[a, b]$, then

$$
\begin{aligned}
& \left|\frac{f(a)+f(b)}{2}-\frac{1}{b-a} \int_{a}^{b} f(x) \mathrm{d} x\right| \\
& \leq \frac{(b-a)}{4}\left[\frac{\left|f^{\prime}(a)\right|^{q}+\left|f^{\prime}(b)\right|^{q}}{2}\right]^{1 / q}
\end{aligned}
$$

and

$$
\begin{aligned}
& \left|f\left(\frac{a+b}{2}\right)-\frac{1}{b-a} \int_{a}^{b} f(x) \mathrm{d} x\right| \\
& \leq \frac{(b-a)}{4}\left[\frac{\left|f^{\prime}(a)\right|^{q}+\left|f^{\prime}(b)\right|^{q}}{2}\right]^{1 / q}
\end{aligned}
$$


$f: I \subset \mathbb{R} \rightarrow \mathbb{R}$. be differentiable on $I^{\circ}, a, b \in I$ with $a<b$, and let $p>1$. If $\left|f^{\prime}(x)\right|^{p /(p-1)}$ is convex on $[a, b]$, then

$$
\begin{aligned}
& \left|\frac{1}{b-a} \int_{a}^{b} f(x) \mathrm{d} x-f\left(\frac{a+b}{2}\right)\right| \\
& \leq \frac{b-a}{16}\left(\frac{4}{p+1}\right)^{1 / p} \times\left\{\left[\left|f^{\prime}(a)\right|^{p /(p-1)}+3\left|f^{\prime}(b)\right|^{p /(p-1)}\right]^{(p-1) / p}\right. \\
& \left.+\left[3\left|f^{\prime}(a)\right|^{p /(p-1)}+\left|f^{\prime}(b)\right|^{p /(p-1)}\right]^{(p-1) / p}\right\}
\end{aligned}
$$

and

$$
\begin{aligned}
& \left|\frac{1}{b-a} \int_{a}^{b} f(x) \mathrm{d} x-f\left(\frac{a+b}{2}\right)\right| \\
& \leq \frac{b-a}{4}\left(\frac{4}{p+1}\right)^{1 / p}\left(\left|f^{\prime}(a)\right|+\left|f^{\prime}(b)\right|\right) .
\end{aligned}
$$

\section{Theorem 1.4 ([6, Theorems 1 and 3]). Let}

$f: I \subset \mathbb{R}_{0} \rightarrow \mathbb{R}$ be differentiable on $I^{\circ}$ and $a, b \in I$ with $a<b$.

(1) If $\left|f^{\prime}(x)\right|^{q}$ is $s$-convex on $[a, b]$ for some fixed $s \in(0,1]$ and $q \geq 1$, then

$$
\begin{aligned}
& \left|\frac{f(a)+f(b)}{2}-\frac{1}{b-a} \int_{a}^{b} f(x) \mathrm{d} x\right| \\
& \leq \frac{(b-a)}{2}\left(\frac{1}{2}\right)^{1-\frac{1}{q}}\left[\frac{2+(1 / 2)^{s}}{(s+1)(s+2)}\right]^{1 / q} \\
& \times\left[\left|f^{\prime}(a)\right|^{q}+\left|f^{\prime}(b)\right|^{q}\right]^{1 / q} .
\end{aligned}
$$

(2) If $\left|f^{\prime}(x)\right|^{q}$ is $s$-convex on $[a, b]$ for some fixed $s \in(0,1]$ and $q>1$, then

$$
\begin{aligned}
& \left|\frac{f(a)+f(b)}{2}-\frac{1}{b-a} \int_{a}^{b} f(x) \mathrm{d} x\right| \\
& \leq \frac{(b-a)}{4}\left(\frac{q-1}{2 q-1}\right)^{1-\frac{1}{q}}\left(\frac{1}{s+1}\right)^{1 / q} \\
& .\left\{\left.|| f^{\prime}(a)\right|^{q}+\left|f^{\prime}\left(\frac{a+b}{2}\right)\right|^{q}\right]^{1 / q} \\
& \left.+\left[\left|f^{\prime}\left(\frac{a+b}{2}\right)\right|^{q}+\left|f^{\prime}(b)\right|^{q}\right]^{1 / q}\right\} \\
& \leq \frac{b-a}{2}\left\{\left[\left|f^{\prime}(a)\right|^{q}+\left|f^{\prime}\left(\frac{a+b}{2}\right)\right|^{q}\right]^{1 / q}\right. \\
& \left.+\left[\left|f^{\prime}\left(\frac{a+b}{2}\right)\right|^{q}+\left|f^{\prime}(b)\right|^{q}\right]^{1 / q}\right\} .
\end{aligned}
$$

Theorem 1.5 ([7, Theorem 2]). Let $f: I \subset \mathbb{R} \rightarrow \mathbb{R}$ be an absolutely continuous function on $I^{\circ}$ such that $f^{\prime \prime \prime} \in L([a, b])$ for $a, b \in I^{\circ}$ with $a, b \in I^{\circ}$. If $\left|f^{\prime \prime \prime}(x)\right|$ is quasi-convex on $[a, b]$, then

$$
\begin{aligned}
&\left|\int_{a}^{b} f(x) \mathrm{d} x-\frac{b-a}{6}\left[f(a)+4 f\left(\frac{a+b}{2}\right)+f(b)\right]\right| \\
& \leq \frac{(b-a)^{4}}{1152}\left[\max \left\{\left|f^{\prime \prime \prime}(a)\right|,\left|f^{\prime \prime \prime}\left(\frac{a+b}{2}\right)\right|\right\}\right. \\
&\left.+\max \left\{\left|f^{\prime \prime \prime}\left(\frac{a+b}{2}\right)\right|,\left|f^{\prime \prime \prime}(b)\right|\right\}\right]
\end{aligned}
$$

In recent years, some other kinds of Hermite-Hadamard type inequalities were created in, for example, [8-17], especially the monographs $[18,19]$, and related references therein.

In this paper, we will find some new inequalities of Hermite-Hadamard type for functions whose third derivatives are $s$-convex and apply these inequalities to discover inequalities for special means.

\section{A Lemma}

For finding some new inequalities of Hermite-Hadamard type for functions whose third derivatives are $s$-convex, we need a simple lemma below.

Lemma 2.1. Let $f: I \subseteq \mathbb{R} \rightarrow \mathbb{R}$ be a three times differentiable function on $I^{\circ}$ with $a, b \in I$ and $a<b$. If $f^{\prime \prime \prime} \in L[a, b]$, then

$$
\begin{aligned}
& \frac{f(a)+f(b)}{2}-\frac{1}{b-a} \int_{a}^{b} f(x) \mathrm{d} x-\frac{b-a}{12}\left[f^{\prime}(b)-f^{\prime}(a)\right] \\
& =\frac{(b-a)^{3}}{12} \int_{0}^{1} t(1-t)(2 t-1) f^{\prime \prime \prime}(t a+(1-t) b) \mathrm{d} t .
\end{aligned}
$$

Proof. By integrating by part, we have

$$
\begin{aligned}
& \int_{0}^{1} t(1-t)(2 t-1) f^{\prime \prime \prime}(t a+(1-t) b) \mathrm{d} t \\
&= \frac{1}{b-a} \int_{0}^{1}\left(-6 t^{2}+6 t-1\right) f^{\prime \prime}(t a+(1-t) b) \mathrm{d} t \\
&=-\frac{\left[f^{\prime}(b)-f^{\prime}(a)\right]}{(b-a)^{2}} \\
&+\frac{1}{(b-a)^{2}} \int_{o}^{1}(-12 t+6) f^{\prime}(t a+(1-t) b) \mathrm{d} t \\
&=-\frac{\left[f^{\prime}(b)-f^{\prime}(a)\right]}{(b-a)^{2}} \\
&-\frac{1}{(b-a)^{3}} \int_{o}^{1}(-12 t+6) \mathrm{d} f(t a+(1-t) b) \\
&=-\frac{\left[f^{\prime}(b)-f^{\prime}(a)\right]}{(b-a)^{2}}+\frac{6[f(a)+f(b)]}{(b-a)^{3}} \\
&-\frac{12}{(b-a)^{3}} \int_{o}^{1} f(t a+(1-t) b) \mathrm{d} t \\
&=-\frac{\left[f^{\prime}(b)-f^{\prime}(a)\right]}{(b-a)^{2}}+\frac{6[f(a)+f(b)]}{(b-a)^{3}} \\
&-\frac{12}{(b-a)^{4}} \int_{a}^{b} f(x) \mathrm{d} x
\end{aligned}
$$


The proof of Lemma 2.1 is complete.

\section{Some New Hermite-Hadamard Type Inequalities}

We now utilize Lemma 2.1, Hölder's inequality, and others to find some new inequalities of Hermite-Hadamard type for functions whose third derivatives are $s$-convex.

Theorem 3.1. Let $f: I \subseteq R_{0} \rightarrow \mathbb{R}$ be a three times differentiable function on $I^{\circ}$ such that $f^{\prime \prime \prime} \in L[a, b]$ for $a, b \in I$ with $a<b$. If $\left|f^{\prime \prime \prime}\right|^{q}$ is $s$-convex on $[a, b]$ for some fixed $s \in(0,1]$ and $q \geq 1$, then

$$
\begin{aligned}
& \left|\frac{f(a)+f(b)}{2}-\frac{1}{b-a} \int_{a}^{b} f(x) \mathrm{d} x-\frac{b-a}{12}\left[f^{\prime}(b)-f^{\prime}(a)\right]\right| \\
& \leq \frac{(b-a)^{3}}{192}\left(\frac{2^{2-s}\left(s+6+2^{s+2} s\right)}{(s+2)(s+3)(s+4)}\right)^{1 / q} \\
& \quad \times\left[\left|f^{\prime \prime \prime}(a)\right|^{q}+\left|f^{\prime \prime \prime}(b)\right|^{q}\right]^{1 / q} .
\end{aligned}
$$

Proof. Since $\left|f^{m}\right|^{q}$ is $s$-convex on $[a, b]$, by Lemma 2.1 and Hölder's inequality, we have

$$
\begin{aligned}
& \left|\frac{f(a)+f(b)}{2}-\frac{1}{b-a} \int_{a}^{b} f(x) \mathrm{d} x-\frac{b-a}{12}\left[f^{\prime}(b)-f^{\prime}(a)\right]\right| \\
& \leq \frac{(b-a)^{3}}{12} \int_{0}^{1} t(1-t)|(2 t-1)|\left|f^{\prime \prime \prime}(t a+(1-t) b)\right| \mathrm{d} t \\
& \leq \frac{(b-a)^{3}}{12} A_{0}^{1-\frac{1}{q}} \\
& \cdot\left[\int_{0}^{1} t(1-t)|(2 t-1)|\left|f^{\prime \prime \prime}(t a+(1-t) b)\right|^{q} \mathrm{~d} t\right]^{1 / q} \\
& \leq \frac{(b-a)^{3}}{12} A_{0}^{1-\frac{1}{q}}\left\{\int _ { 0 } ^ { 1 } t ( 1 - t ) | ( 2 t - 1 ) | \left[t^{s}\left|f^{\prime \prime \prime}(a)\right|^{q}\right.\right. \\
& \left.\left.+(1-t)^{S}\left|f^{\prime \prime \prime}(b)\right|^{q}\right] \mathrm{~d} t\right\}^{1 / q},
\end{aligned}
$$

where

$$
A_{0}=\int_{0}^{1} t(1-t)|(2 t-1)| \mathrm{d} t=\frac{1}{16}
$$

and

$$
\begin{aligned}
A_{s} & =\int_{0}^{1} t(1-t)|(2 t-1)| t^{s} \mathrm{~d} t \\
& =\int_{0}^{1} t(1-t)|(2 t-1)|(1-t)^{s} \mathrm{~d} t \\
& =\frac{6+s+2^{s+2} s}{2^{s+2}(s+2)(s+3)(s+4)} .
\end{aligned}
$$

Thus, we have

$$
\begin{aligned}
& \left|\frac{f(a)+f(b)}{2}-\frac{1}{b-a} \int_{a}^{b} f(x) \mathrm{d} x-\frac{b-a}{12}\left[f^{\prime}(b)-f^{\prime}(a)\right]\right| \\
& \leq \frac{(b-a)^{3}}{12}\left(\frac{1}{16}\right)^{1-\frac{1}{q}}\left(\frac{s+6+2^{s+2} s}{2^{s+2}(s+2)(s+3)(s+4)}\right)^{1 / q} \\
& \times\left[\left|f^{\prime \prime \prime}(a)\right|^{q}+\left|f^{\prime \prime \prime}(b)\right|^{q}\right]^{1 / q} \\
& =\frac{(b-a)^{3}}{192}\left(\frac{2^{2-s}\left(s+6+2^{s+2} s\right)}{(s+2)(s+3)(s+4)}\right)^{1 / q} \\
& \times\left[\left|f^{\prime \prime \prime}(a)\right|^{q}+\left|f^{\prime \prime \prime}(b)\right|^{q}\right]^{1 / q} .
\end{aligned}
$$

The proof of Theorem 3.1 is complete.

Corollary 3.1.1. Under conditions of Theorem 3.1,

1) if $s=1$, then

$$
\begin{aligned}
& \left|\frac{f(a)+f(b)}{2}-\frac{1}{b-a} \int_{a}^{b} f(x) \mathrm{d} x-\frac{b-a}{12}\left[f^{\prime}(b)-f^{\prime}(a)\right]\right| \\
& \leq \frac{(b-a)^{3}}{192}\left(\frac{1}{2}\right)^{1 / q}\left[\left|f^{\prime \prime \prime}(a)\right|^{q}+\left|f^{\prime \prime \prime}(b)\right|^{q}\right]^{1 / q} ;
\end{aligned}
$$

2) if $q=s=1$, then

$$
\begin{aligned}
& \left|\frac{f(a)+f(b)}{2}-\frac{1}{b-a} \int_{a}^{b} f(x) \mathrm{d} x-\frac{b-a}{12}\left[f^{\prime}(b)-f^{\prime}(a)\right]\right| \\
& \leq \frac{(b-a)^{3}}{384}\left[\left|f^{\prime \prime \prime}(a)\right|+\left|f^{\prime \prime \prime}(b)\right|\right] .
\end{aligned}
$$

Theorem 3.2. Let $f: I \subseteq R_{0} \rightarrow \mathbb{R}$ be a three times differentiable function on $I^{\circ}$ such that $f^{\prime \prime \prime} \in L[a, b]$ for $a, b \in I$ with $a<b$. If $\left|f^{\prime \prime \prime}\right|^{q}$ is $s$-convex on $[a, b]$ for some fixed $s \in(0,1]$ and $q>1$, then

$$
\begin{aligned}
& \left|\frac{f(a)+f(b)}{2}-\frac{1}{b-a} \int_{a}^{b} f(x) \mathrm{d} x-\frac{b-a}{12}\left[f^{\prime}(b)-f^{\prime}(a)\right]\right| \\
& \leq \frac{(b-a)^{3}}{96}\left(\frac{1}{p+1}\right)^{1 / p}\left(\frac{2^{1-s}\left(s 2^{s}+1\right)}{(s+1)(s+2)}\right)^{1 / q}
\end{aligned}
$$$$
\cdot\left[\left|f^{\prime \prime \prime}(a)\right|^{q}+\left|f^{\prime \prime \prime}(b)\right|^{q}\right]^{1 / q},
$$

where $\frac{1}{q}+\frac{1}{p}=1$.

Proof. Using Lemma 2.1, the $s$-convexity of $\left|f^{\prime m^{\prime}}\right|^{q}$ on $[a, b]$, and Hölder's integral inequality yields 


$$
\begin{aligned}
& \left|\frac{f(a)+f(b)}{2}-\frac{1}{b-a} \int_{a}^{b} f(x) \mathrm{d} x-\frac{b-a}{12}\left[f^{\prime}(b)-f^{\prime}(a)\right]\right| \\
& \leq \frac{(b-a)^{3}}{12} \int_{0}^{1} t(1-t)|(2 t-1)|\left|f^{\prime \prime \prime}(t a+(1-t) b)\right| \mathrm{d} t \\
& \leq \frac{(b-a)^{3}}{12} B^{1 / p}\left[\int_{0}^{1}|2 t-1|\left|f^{\prime \prime \prime}(t a+(1-t) b)\right|^{q} \mathrm{~d} t\right]^{1 / q} \\
& \leq \frac{(b-a)^{3}}{12} B^{1 / p} \\
& \cdot\left\{\int_{0}^{1}|2 t-1|\left[t^{s}\left|f^{\prime \prime \prime}(a)\right|^{q}+(1-t)^{s}\left|f^{\prime^{\prime \prime}}(b)\right|^{q}\right] \mathrm{d} t\right\}^{1 / q},
\end{aligned}
$$

where an easy calculation gives

$$
\begin{aligned}
B & =\int_{0}^{1} t^{p}(1-t)^{p}|2 t-1| \mathrm{d} t \\
& =\frac{1}{2^{2 p+1}(p+1)}
\end{aligned}
$$

and

$$
\begin{aligned}
\int_{0}^{1} t^{s}|2 t-1| \mathrm{d} t & =\int_{0}^{1}(1-t)^{s}|2 t-1| \mathrm{d} t \\
& =\frac{s 2^{s}+1}{2^{s}(s+1)(s+2)} .
\end{aligned}
$$

Substituting Equations (3.4) and (3.5) into the above inequality results in the inequality (3.3). The proof of Theorem 3.2 is complete.

Corollary 3.2.1. Under conditions of Theorem 3.2, if $s=1$, then

$$
\begin{aligned}
& \left|\frac{f(a)+f(b)}{2}-\frac{1}{b-a} \int_{a}^{b} f(x) \mathrm{d} x-\frac{b-a}{12}\left[f^{\prime}(b)-f^{\prime}(a)\right]\right| \\
& \leq \frac{(b-a)^{3}}{96}\left(\frac{1}{p+1}\right)^{1 / p}\left(\frac{1}{2}\right)^{1 / q}\left[\left|f^{\prime \prime \prime}(a)\right|^{q}+\left|f^{\prime \prime \prime}(b)\right|^{q}\right]^{1 / q} .
\end{aligned}
$$

Theorem 3.3. Under conditions of Theorem 3.2, we have

$$
\begin{aligned}
& \left|\frac{f(a)+f(b)}{2}-\frac{1}{b-a} \int_{a}^{b} f(x) \mathrm{d} x-\frac{b-a}{12}\left[f^{\prime}(b)-f^{\prime}(a)\right]\right| \\
& \leq \frac{(b-a)^{3}}{24}\left(\frac{1}{(p+1)(p+3)}\right)^{1 / p}\left(\frac{2}{(s+2)(s+3)}\right)^{1 / q} \\
& \quad \times\left[\left|f^{\prime \prime \prime}(a)\right|^{q}+\left|f^{\prime \prime \prime}(b)\right|^{q}\right]^{1 / q} .
\end{aligned}
$$

Proof. Making use of Lemma 2.1, the $s$-convexity of $\left|f^{\prime \prime \prime}\right|^{q}$ on $[a, b]$, and Hölder's integral inequality leads to

$$
\begin{aligned}
& \left|\frac{f(a)+f(b)}{2}-\frac{1}{b-a} \int_{a}^{b} f(x) \mathrm{d} x-\frac{b-a}{12}\left[f^{\prime}(b)-f^{\prime}(a)\right]\right| \\
& \leq \frac{(b-a)^{3}}{12} C^{1 / p}\left[\int_{0}^{1} t(1-t)\left|f^{\prime \prime \prime}(t a+(1-t) b)\right|^{q} \mathrm{~d} t\right]^{1 / q} \\
& \leq \frac{(b-a)^{3}}{12} C^{1 / p} \\
& \cdot\left\{\int_{0}^{1} t(1-t)\left[t^{s}\left|f^{\prime \prime \prime}(a)\right|^{q}+(1-t)^{s}\left|f^{\prime \prime \prime}(b)\right|^{q}\right] \mathrm{d} t\right\}^{1 / q},
\end{aligned}
$$

where

$$
C=\int_{0}^{1} t(1-t)|(2 t-1)|^{p} \mathrm{~d} t=\frac{1}{2(p+1)(p+3)}
$$

and

$$
\int_{0}^{1} t^{s+1}(1-t) \mathrm{d} t=\int_{0}^{1} t(1-t)^{s+1} \mathrm{~d} t=\frac{1}{(s+2)(s+3)} .
$$

Substituting Equations (3.7) and (3.8) into the above inequality derives the inequality (3.6). The proof of Theorem 3.3 is complete.

Corollary 3.3.1. Under conditions of Theorem 3.3, if $s$ $=1$, then

$$
\begin{aligned}
& \left|\frac{f(a)+f(b)}{2}-\frac{1}{b-a} \int_{a}^{b} f(x) \mathrm{d} x-\frac{b-a}{12}\left[f^{\prime}(b)-f^{\prime}(a)\right]\right| \\
& \leq \frac{(b-a)^{3}}{24}\left(\frac{1}{(p+1)(p+3)}\right)^{1 / p}\left(\frac{1}{6}\right)^{1 / q} \\
& \cdot\left[\left|f^{\prime \prime \prime}(a)\right|^{q}+\left|f^{\prime \prime \prime}(b)\right|^{q}\right]^{1 / q} .
\end{aligned}
$$

Theorem 3.4. Under conditions of Theorem 3.2, we have

$$
\begin{aligned}
& \left|\frac{f(a)+f(b)}{2}-\frac{1}{b-a} \int_{a}^{b} f(x) \mathrm{d} x-\frac{b-a}{12}\left[f^{\prime}(b)-f^{\prime}(a)\right]\right| \\
& \leq \frac{(b-a)^{3}}{48}\left(\frac{5+2^{p+1}(p-1)+p}{(p+1)(p+2)(p+3)}\right)^{1 / p} \\
& \left(\frac{1}{2^{s}(s+1)(s+2)(s+3)}\right)^{1 / q} \\
& \times \min \left\{\left[\left(5+2^{s+1}(s-1)+s\right)\left|f^{\prime \prime \prime}(a)\right|^{q}\right]\right. \\
& \left.+\left(2^{s+1}(s+1)^{2}+s+1\right)\left|f^{\prime \prime \prime}(b)\right|^{q}\right]^{1 / q}, \\
& \quad\left[\left(2^{s+1}(s+1)^{2}+s+1\right)\left|f^{\prime \prime \prime}(a)\right|^{q}\right. \\
& \left.\left.+\left(5+2^{s+1}(s-1)+s\right)\left|f^{\prime \prime \prime}(b)\right|^{q}\right]^{1 / q}\right\}
\end{aligned}
$$

Proof. Since $\left|f^{\prime \prime \prime}\right|^{q}$ is $s$-convex on $[a, b]$, by Lemma 
2.1 and Hölder's inequality, we have

$\left|\frac{f(a)+f(b)}{2}-\frac{1}{b-a} \int_{a}^{b} f(x) \mathrm{d} x-\frac{b-a}{12}\left[f^{\prime}(b)-f^{\prime}(a)\right]\right|$

$\leq \frac{(b-a)^{3}}{12} \int_{0}^{1} t(1-t)|2 t-1|\left|f^{\prime \prime \prime}(t a+(1-t) b)\right| \mathrm{d} t$

$\leq \frac{(b-a)^{3}}{12} D^{1 / p}$

$\left\{\int_{0}^{1} t(1-t)|2 t-1|\left[t^{s}\left|f^{\prime \prime \prime}(a)\right|^{q}+(1-t)^{s}\left|f^{\prime \prime \prime}(b)\right|^{q}\right] \mathrm{d} t\right\}^{1 / q}$

and

$\left|\frac{f(a)+f(b)}{2}-\frac{1}{b-a} \int_{a}^{b} f(x) \mathrm{d} x-\frac{b-a}{12}\left[f^{\prime}(b)-f^{\prime}(a)\right]\right|$ $\leq \frac{(b-a)^{3}}{12} D^{1 / p}$

$\cdot\left\{\int_{0}^{1} t|2 t-1|\left[t^{s}\left|f^{\prime \prime \prime}(a)\right|^{q}+(1-t)^{s}\left|f^{\prime \prime \prime}(b)\right|^{q}\right] \mathrm{d} t\right\}^{1 / q}$

where a straightforward computation gives

$$
\begin{aligned}
& D=\int_{0}^{1} t^{P}(1-t)|2 t-1| \mathrm{d} t=\frac{5+2^{p+1}(p-1)+p}{2^{p+1}(p+1)(p+2)(p+3)}, \\
& \int_{0}^{1} t(1-t)^{p}|2 t-1| \mathrm{d} t=\frac{5+2^{p+1}(p-1)+p}{2^{p+1}(p+1)(p+2)(p+3)}, \\
& \int_{0}^{1}(1-t)^{s+1}|2 t-1| \mathrm{d} t=\frac{2^{s+1}(s+1)+1}{2^{s+1}(s+2)(s+3)}, \\
& \int_{0}^{1}(t)^{s+1}|2 t-1| \mathrm{d} t=\frac{2^{s+1}(s+1)+1}{2^{s+1}(s+2)(s+3)} .
\end{aligned}
$$

Substituting these equalities into the above inequality brings out the inequality (3.10). The proof of Theorem 3.4 is complete.

Corollary 3.4.1. Under conditions of Theorem 3.4, if $s=1$, then

$$
\begin{aligned}
& \left|\frac{f(a)+f(b)}{2}-\frac{1}{b-a} \int_{a}^{b} f(x) \mathrm{d} x-\frac{b-a}{12}\left[f^{\prime}(b)-f^{\prime}(a)\right]\right| \\
& \leq \frac{(b-a)^{3}}{48}\left(\frac{5+2^{p+1}(p-1)+p}{(p+1)(p+2)(p+3)}\right)^{1 / p}\left(\frac{1}{8}\right)^{1 / q} \\
& \quad \times \min \left\{\left[\left|f^{\prime \prime \prime}(a)\right|^{q}+3\left|f^{\prime \prime \prime}(b)\right|^{q}\right]^{1 / q},\right. \\
& \left.\quad\left[3\left|f^{\prime \prime \prime}(a)\right|^{q}+\left|f^{\prime \prime \prime}(b)\right|^{q}\right]^{1 / q}\right\} .
\end{aligned}
$$

\section{Applications to Special Means}

For positive numbers $a>0$ and $b>0$, define

$$
A(a, b)=\frac{a+b}{2}
$$

and

$$
L_{r}(a, b)=\left\{\begin{array}{cc}
{\left[\frac{b^{r+1}-a^{r+1}}{(r+1)(b-a)}\right]^{1 / r},} & r \neq-1,0 ; \\
\frac{b-a}{\ln b-\ln a} & , \quad r=-1 ; \\
\frac{1}{e}\left(\frac{b^{b}}{a^{a}}\right)^{1 /(b-a)} & , \quad r=0 .
\end{array}\right.
$$

It is well known that $A$ and $L_{r}$ are respectively called the arithmetic and generalized logarithmic means of two positive number $a$ and $b$.

Now we are in a position to construct some inequalities for special means $A$ and $L_{r}$ by applying the above established inequalities of Hermite-Hadamard type.

Let

$$
f(x)=\frac{x^{s+3}}{(s+1)(s+2)(s+3)}
$$

for $0<s \leq 1$ and $x>0$. Since $f^{\prime \prime \prime}(x)=x^{s}$ and $(\lambda x+(1-\lambda) y)^{s} \leq \lambda^{s} x^{s}+(1-\lambda)^{s} y^{s}$

for $x, y>0$ and $\lambda \in[0,1]$, then $f^{\prime \prime \prime}(x)=x^{s}$ is $s$-convex function on $\mathbb{R}_{0}$ and

$$
\begin{gathered}
\frac{f(a)+f(b)}{2}=\frac{1}{(s+1)(s+2)(s+3)} A\left(a^{s+3}, b^{s+3}\right), \\
\frac{1}{b-a} \int_{a}^{b} f(x) \mathrm{d} x=\frac{1}{(s+1)(s+2)(s+3)} L_{s+3}^{s+3}\left(a^{s+4}, b^{s+4}\right), \\
f^{\prime}(b)-f^{\prime}(a)=\frac{1}{12(s+1)} L_{s+1}^{s+1}\left(a^{s+2}, b^{s+2}\right) .
\end{gathered}
$$

Applying the function (4.3) to Theorems 3.1 to 3.3 immediately leads to the following inequalities involving special means $A$ and $L_{r}$.

Theorem 4.1. Let $b>a>0,0<s \leq 1$, and $q \geq 1$. Then

$$
\begin{aligned}
& \mid 12 A\left(a^{s+3}, b^{s+3}\right)-12 L_{s+3}^{s+3}\left(a^{s+4}, b^{s+4}\right) \\
& -(b-a)^{2}(s+2)(s+3) L_{s+1}^{s+1}\left(a^{s+2}, b^{s+2}\right) \mid \\
& \leq \frac{(b-a)^{3}(s+1)}{16}[(s+2)(s+3)]^{1-\frac{1}{q}} \\
& \quad \times\left[\frac{2^{3-s}\left(s+6+2^{s+2} s\right)}{s+4}\right]^{1 / q} \\
& \quad \times A^{1 / q}\left(a^{s q}, b^{s q}\right) .
\end{aligned}
$$


Theorem 4.2. For $b>a>0,0<s \leq 1$, and $q>1$, we have

$$
\begin{aligned}
& \mid 12 A\left(a^{s+3}, b^{s+3}\right)-12 L_{s+3}^{s+3}\left(a^{s+4}, b^{s+4}\right) \\
& -(b-a)^{2}(s+2)(s+3) L_{s+1}^{s+1}\left(a^{s+2}, b^{s+2}\right) \mid \\
& \leq \frac{(b-a)^{3}(s+3)}{8}\left(\frac{(s+1)(s+2)}{p+1}\right)^{1 / p} \\
& \quad \times\left[2^{2-s}\left(s 2^{s}+1\right)\right]^{1 / q} A^{1 / q}\left(a^{s q}, b^{s q}\right) .
\end{aligned}
$$

Theorem 4.3. For $b>a>0,0<s \leq 1$, and $q>1$, we have

$$
\begin{aligned}
& \mid 12 A\left(a^{s+3}, b^{s+3}\right)-12 L_{s+3}^{s+3}\left(a^{s+4}, b^{s+4}\right) \\
& -(b-a)^{2}(s+2)(s+3) L_{s+1}^{s+1}\left(a^{s+2}, b^{s+2}\right) \mid \\
& \leq 2(b-a)^{3}(s+1)\left[\frac{(s+2)(s+3)}{4(p+1)(p+3)}\right]^{1 / p} A^{1 / q}\left(a^{s q}, b^{s q}\right) .
\end{aligned}
$$

\section{Acknowledgements}

The first author was supported by Science Research Funding of Inner Mongolia University for Nationalities under Grant No. NMD1103.

\section{REFERENCES}

[1] S. S. Dragomir, J. Pecaric and L.-E. Persson, "Some Inequalities of Hadamard Type," Soochow Journal of Mathematics, Vol. 21, No. 3, 1995, pp. 335-341.

[2] H. Hudzik and L. Maligranda, "Some Remarks on $s$-Convex Functions," Aequationes Mathematicae, Vol. 48, No. 1, 1994, pp. 100-111.

[3] S. S. Dragomir and R. P. Agarwal, "Two Inequalities for Differentiable Mappings and Applications to Special Means of Real Numbers and to Trapezoidal Formula," Applied Mathematics Letters, Vol. 11, No. 5, 1998, pp. 91-95. doi:10.1016/S0893-9659(98)00086-X

[4] C. E. M. Pearce and J. Pečarić, "Inequalities for Differentiable Mappings with Application to Special Means and Quadrature Formulae," Applied Mathematics Letters, Vol. 13, No. 2, 2000, pp. 51-55. doi:10.1016/S0893-9659(99)00164-0

[5] U. S. Kirmaci, "Inequalities for Differentiable Mappings and Applications to Special Means of Real Numbers and to Midpoint Formula," Applied Mathematics and Computation, Vol. 147, No. 1, 2004, pp. 137-146. doi:10.1016/S0096-3003(02)00657-4

[6] U. S. Kirmaci, M. K. Bakula, M. E. Ozdemir and J. Pecaric, "Hadamard-Type Inequalities for $s$-Convex Functions," Applied Mathematics and Computation, Vol. 193, No. 1, 2007, pp. 26-35. doi:10.1016/j.amc.2007.03.030
[7] M. Alomari and S. Hussain, "Two Inequalities of Simpson Type for Quasi-Convex Functions and Applications," Applied Mathematics E-Notes, Vol. 11, 2011, pp. 110117.

[8] R.-F. Bai, F. Qi and B.-Y. Xi, "Hermite-Hadamard Type Inequalities for the $m$ - and $(\alpha, m)$-Logarithmically Convex Functions," Filomat, Vol. 27, No. 1, 2013, 1-7.

[9] S.-P. Bai, S.-H. Wang and F. Qi, "Some Hermite-Hadamard Type Inequalities for $n$-Time Differentiable $(\alpha$, $m$ )-Convex Functions," Journal of Inequalities and Applications, 2013, in Press.

[10] W.-D. Jiang, D.-W. Niu, Y. Hua and F. Qi, "Generalizations of Hermite-Hadamard Inequality to $n$-Time Differentiable Functions Which Are $s$-Convex in the Second Sense," Analysis (Munich), Vol. 32, No. 3, 2012, pp. 209220. doi:10.1524/anly.2012.1161

[11] F. Qi, Z.-L. Wei and Q. Yang, "Generalizations and Refinements of Hermite-Hadamard's Inequality," The Rocky Mountain Journal of Mathematics, Vol. 35, No. 1, 2005, pp. 235-251. doi:10.1216/rmjm/1181069779

[12] S.-H. Wang, B.-Y. Xi and F. Qi, "On Hermite-Hadamard Type Inequalities for $(\alpha, m)$-Convex Functions," International Journal of Open Problems in Computer Science and Mathematics, Vol. 5, No. 4, 2012, in Press.

[13] S.-H. Wang, B.-Y. Xi and F. Qi, "Some New Inequalities of Hermite-Hadamard Type for $n$-Time Differentiable Functions Which Are $m$-Convex," Analysis (Munich), Vol. 32, No. 3, 2012, pp. 247-262. doi:10.1524/anly.2012.1167

[14] B.-Y. Xi, R.-F. Bai and F. Qi, "Hermite-Hadamard Type Inequalities for the $m$ - and $(\alpha ; m)$-Geometrically Convex Functions," Aequationes Mathematicae, 2012, in Press. doi:10.1007/s00010-011-0114-X

[15] B.-Y. Xi and F. Qi, "Some Hermite-Hadamard Type Inequalities for Differentiable Convex Functions and Applications," Hacettepe Journal of Mathematics and Statistics, Vol. 42, 2013, in Press.

[16] B.-Y. Xi and F. Qi, "Some Integral Inequalities of Hermite-Hadamard Type for Convex Functions with Applications to Means," Journal of Function Spaces and Applications, Vol. 2012, 2012, 14 pp. doi:10.1155/2012/980438

[17] T.-Y. Zhang, A.-P. Ji and F. Qi, "On Integral Inequalities of Hermite-Hadamard Type for $s$-Geometrically Convex Functions," Abstract and Applied Analysis, Vol. 2012, 2012, 15 pp. doi: $10.1155 / 2012 / 560586$

[18] S. S. Dragomir and C. E. M. Pearce, "Selected Topics on Hermite-Hadamard Type Inequalities and Applications," RGMIA Monographs, Victoria University, Melbourne, 2000.

[19] C. P. Niculescu and L.-E. Persson, "Convex Functions and Their Applications: A Contemporary Approach (CMS Books in Mathematics)," Springer-Verlag, New York, 2005. 